\title{
Computing Deformable Image Registration by Inverse Multiquadric Demons Algorithm
}

\author{
S. M. Wong, K. S. Ng, and T. S. Li
}

\begin{abstract}
Radiation therapy is the mainstay of cancer treatment. However, significant weight loss and tumor shrinkage during treatment could affect the medical outcome. Advanced forms of adaptive radiotherapy use deformable image registration (DIR) accounting for such anatomical changes. Accurately defining the exact location and extent of the target with respect to surrounding organs at risk are difficult tasks. DIR model is commonly used as an alternative to measure how the tumor changes in size and location. In this paper, a reciprocal Multiquadric radial basis function approximation method is incorporated with the Demons algorithm for solving the DIR model. The proposed scheme could enhance the accuracy and efficiency of the deformable image registration. The numerical performance is compared with the classical DIR Demons algorithms. The result demonstrated that the combination of the reciprocal Multiquadric approximation and the Demons algorithm is useful in image registration.
\end{abstract}

Index Terms-Deformable image registration, Demons algorithm, radial basis functions.

\section{INTRODUCTION}

Cancer has been the leading cause of death over the last decades. Majority of cancer patients are treated by the external beam radiotherapy treatment. In order to inhibit the tumor growth and minimize the side effects to the patient, confining the treatment to the target region and avoiding damage to healthy organs are important issues during the treatment process. This can be done by Image Guided Radiation Therapy (IGRT).

The basic deformable image registration (DIR) is an optimization method used to delineate the clinically critical objects in computed tomography image obtained during the radiation treatment. The first deformable image registration method was proposed by Broit [1] in 1981 .

The study of DIR has last for more than 30 years in the discipline of biomedical engineering. It has become an important tool in radiotherapy treatment. Brain surgery and neurosciences have been the driving applications for developing an abundant number of techniques as discussed in [2] and [3]. Despite the significant progress that has been made, deformable registration remains a challenging problem in the field of radiotherapy. The original DIR model was developed to describe the diffusion of spatial transformation between the profile of tumor at $i^{\text {th }}$ treatment and the corresponding profile of tumor at $(i+1)^{\text {th }}$ treatment. The volumetric analysis and the

Manuscript received June 15, 2013; revised July 20, 2013. This work was supported by the Research and Development Fund of the Open University of Hong Kong, No. 2010/2.1.

The Authors are with the School of Science and Technology, the Open University of Hong Kong (e-mail: anwong@ouhk.edu.hk, tsli@ouhk.edu.hk, dng@ouhk.edu.hk ). positional changes of the tumor can be monitored and adaptive medical strategies can be used during the course of treatment. The DIR model is an ill-posed problem because there is generally no unique solution to an image registration problem. Image registration can be defined as finding the function $g$ in the mapping between two images $I_{1}$ and $I_{2}$ as shown in (1).

$$
I_{2}(x, y)=g\left[I_{1}(x, y)\right],
$$

where $I_{1}$ is called the moving image and $I_{2}$ is called the static image. The images $I_{1}$ and $I_{2}$ can be thought of as $\mathbb{R}^{2} \rightarrow \mathbb{R}^{2}$ mappings from two dimensional coordinates to image intensities. An example of 2D coordinates mapping system is illustrated in Fig. 1.

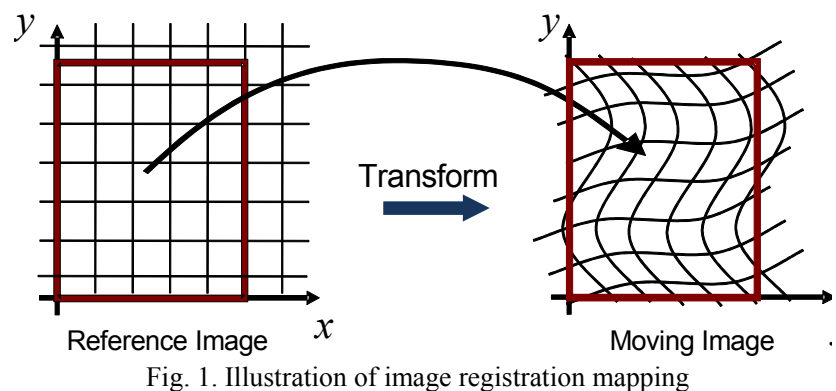

The DIR is modelled as a two-dimensional homogeneous infinite elastic medium. It is assumed that external forces are applied at every control point, where the strengths, directions and influence areas of the forces as well as the positions of the control points are considered.

The finite element method (FEM) and finite difference method (FDM) have been an important tool in biomedical engineering and commonly used to solve the DIR model. Among the discretization methods, the techniques of FEM have been demonstrated to be the best choice for solving partial differential equations. However, the drawback of FEM-based method is its higher computational complexity. This disadvantage arises from the fact that FEM requires a background mesh of the target domain be defined in advance. The generating and regenerating of a mesh could be a great burden especially for handling complicated problems. The efforts of seeking meshless methods to avoid the burden of mesh generation began in 1970's by Hardy [4].

The development of meshless radial basis function (RBF) computational methods over the last decade had been proven to be effective in solving various types of differential equations. RBFs were first used in neural network and later as bases for multivariate interpolation.

Recently, radial basis function (RBF) methods have been recognized as more suitable for solving problems with complicated domains. It has attracted much attention in 
solving medical image registration problems due to its flexibility and efficiency. The RBF approximation methods have major advantages over the finite element method. One of these advantages is that it does not require the construction of an underlying mesh, and therefore it can deal with complicated or irregular domains more easily.

The thin plate spline (TPS) is a particular type of radial basis functions derived from the minimization of a smoothness measure based on the partial derivatives of the transformation as reported by Bookstein [3]. Lately Osorio [5] combined successfully the automatic feature correspondence optimization for non-rigid registration with $\mathrm{RBF}$ interpolation in radiotherapy.

This study adopted the core component of deformable registration model called Demons algorithm developed by Thirion [6]. In order to improve the Demons algorithm, we incorporate a class of RBFs called the reciprocal Multiquadric function with the Demons algorithm. The proposed algorithm is used to simulate the change of deformation registration image across the sectional tumor. A numerical case study based on a prostate registration image is presented in this study. The numerical performances are compared with results obtained by the classic DIR Demons algorithm.

\section{Deformable Image Registration Model}

The governing equations of the deformation field are based on Navier nonlinear elastic model. Let $\mathbf{D}=\left[\mathrm{u}_{\mathrm{x}}, \mathrm{u}_{\mathrm{y}}\right]^{\mathbf{T}}$ be the incremental displacement for the moving image, the basic mathematical formation is given by (2).

$$
\mathbf{D}=\frac{(\boldsymbol{m}-\boldsymbol{s}) \nabla \boldsymbol{s}}{|\nabla \boldsymbol{s}|^{2}+|(\boldsymbol{m}-\boldsymbol{s})|^{2}}
$$

where $\mathbf{m}$ is moving image and $\mathbf{s}$ is the static image, $(\boldsymbol{m}-\boldsymbol{s})$ are the differential forces between the moving image and the static image. The operator $\nabla \mathbf{s}$ is the gradients in each nodal point $(x, y)$ of the static image $\mathbf{s}$ and is defined by

$$
\nabla \mathbf{s}(x, y)=\left(\frac{\partial s(x, y)}{\partial x}, \frac{\partial s(x, y)}{\partial y}\right)
$$

Equation (2) can be rearranged as a homogenous equation as in (3).

$$
\mathbf{D}\left(|\nabla \boldsymbol{s}|^{2}+|(\boldsymbol{m}-\boldsymbol{s})|^{2}\right)-(\boldsymbol{m}-\boldsymbol{s}) \nabla \boldsymbol{s}=\mathbf{0} .
$$

In mathematics, the solution of spatial discretization of the differential equations in (3) can be approximated by numerical methods such as the finite difference and finite element schemes. These approximation methods generally require an underlying grid system or mesh configuration to support the computations. Mesh generation could be extremely difficult and troublesome if the geometry of the target region is irregular.

In this paper, a radial basis function (RBF) method is used to solve the deformable image registration model. RBF is a truly meshless numerical scheme. As there is no need to have a mesh, so the RBF method is particularly good in handling problems with large deformations.

In order to evaluate the computational results of RBF method, the classical iterative formula of Demons algorithm is also set up as a reference model in this study. The corresponding computational results of the Demons algorithm and RBF Demons algorithm are illustrated and discussed.

\section{FORMULATION OF DEMON'S ALGORITHM}

The original Demons algorithm devised by Thirion [6] is a classical optimization scheme to solve the deformable image registration. The displacement of deviator $\mathbf{D}$ between the moving image ( $\mathbf{m})$ and the reference image (s) is computed by using the iterative formula as given in (4).

$$
\mathbf{D}_{i, j}^{n}=\mathbf{D}_{i, j}^{n-1}-\frac{\left(\mathbf{m}_{i, j}^{n-1}-\mathbf{s}_{i, j}^{0}\right) \nabla \mathbf{s}_{i, j}^{0}}{\left|\nabla \mathbf{s}_{i, j}^{0}\right|^{2}+\left|\left(\mathbf{m}_{i, j}^{n-1}-\mathbf{s}_{i, j}^{0}\right)\right|^{2}},
$$

for $i, j=1,2,3, \cdots, N$. This iterative equation is subject to the following initial conditions:

$$
\left\{\begin{array}{c}
\mathbf{D}_{i, j}^{0}=\mathbf{0} \\
\mathbf{m}_{i, j}^{0}=\widehat{\mathbf{m}}_{i j}, \\
\mathbf{s}_{i, j}^{0}=\hat{\mathbf{s}}_{i j},
\end{array}\right.
$$

where $\widehat{\mathbf{m}}_{\boldsymbol{i} \boldsymbol{j}}$ and $\hat{\mathbf{s}}_{\boldsymbol{i} \boldsymbol{j}}$ are intensities of the original static and move images at the corresponding pixel.

Pennec [7] in 1999 improved the Demons algorithm by take into consideration a weighted factor $\omega$. It attempted to normalize the relations between the moving and static image. The corresponding improved Demon's algorithm is given in (5).

$$
\mathbf{D}_{i, j}^{n}=\mathbf{D}_{i, j}^{n-1}-\left(\begin{array}{c}
\frac{\left(\mathbf{m}_{i, j}^{n-1}-\mathbf{s}_{i, j}^{0}\right) \nabla \mathbf{s}_{i, j}^{0}}{\left|\nabla \mathbf{s}_{i, j}^{0}\right|^{2}+\omega^{2}\left|\left(\mathbf{m}_{i, j}^{n-1}-\mathbf{s}_{i, j}^{0}\right)\right|^{2}}+ \\
\frac{\left(\mathbf{m}_{i, j}^{n-1}-\mathbf{s}_{i, j}^{0}\right) \nabla \mathbf{m}_{i, j}^{0}}{\left|\nabla \mathbf{s}_{i, j}^{0}\right|^{2}+\omega^{2}\left|\left(\mathbf{m}_{i, j}^{n-1}-\mathbf{s}_{i, j}^{0}\right)\right|^{2}}
\end{array}\right) .
$$

In the above algorithms, the registered image $\mathbf{m}_{i, j}^{n}$ in the n-th iterations is computed by inputting $\mathbf{D}_{i, j}^{n-1}$ and $\mathbf{m}_{i, j}^{(n-1)}$ as defined in (6).

$$
\mathbf{m}_{i, j}^{n}=\mathbf{m}_{i, j}^{(n-1)}+\mathbf{D}_{i, j}^{n-1} \nabla \mathbf{s}_{i, j}^{0}
$$

In the algorithm, the distance of $\nabla \mathrm{x}$ and $\nabla \mathrm{y}$ between two neighbourhood pixels is taken to be 1 . The gradients $\nabla \mathbf{s}_{i, j}^{0}$ of static image are $\mathrm{N} \times \mathrm{N}$ coefficient matrix and are calculated once using (7) and (8).

$$
\begin{aligned}
\nabla \mathbf{s}_{x}^{0} & =\left[\begin{array}{cccc}
\mathrm{s}_{1,2}^{0}-\mathrm{s}_{1,1}^{0} & \cdots & \mathrm{s}_{1, N}^{0}-\mathrm{s}_{1, N-1}^{0} & \mathrm{~s}_{1, N}^{0}-\mathrm{s}_{1, N-1}^{0} \\
\vdots & \ddots & \vdots & \vdots \\
\mathrm{s}_{N, 2}^{0}-\mathrm{s}_{N, 1}^{0} & \cdots & \mathrm{s}_{N, N}^{0}-\mathrm{s}_{N, N-1}^{0} & \mathrm{~s}_{N, N}^{0}-\mathrm{s}_{N, N-1}^{0}
\end{array}\right] \\
\nabla \mathbf{s}_{\boldsymbol{y}}^{0} & =\left[\begin{array}{cccc}
\mathrm{s}_{2,1}^{0}-\mathrm{s}_{1,1}^{0} & \mathrm{~s}_{2,2}^{0}-\mathrm{s}_{2,1}^{0} & \cdots & \mathrm{s}_{2, N}^{0}-\mathrm{s}_{1, N-1}^{0} \\
\vdots & \vdots & \ddots & \vdots \\
\mathrm{s}_{N, 1}^{0}-\mathrm{s}_{N-1,1}^{0} & \mathrm{~s}_{N, 2}^{0}-\mathrm{s}_{N-1,2}^{0} & \cdots & \mathrm{s}_{N, N}^{0}-\mathrm{s}_{N-1, N}^{0} \\
\mathrm{~s}_{N, 1}^{0}-\mathrm{s}_{N-1,1}^{0} & \mathrm{~s}_{N, 2}^{0}-\mathrm{s}_{N-1,2}^{0} & \cdots & \mathrm{s}_{N, N}^{0}-\mathrm{s}_{N-1, N}^{0}
\end{array}\right]
\end{aligned}
$$


The registered image $\mathbf{m}_{i, j}^{n}$ can be determined after $n$ iterations. The iterative stopping criterion is set as in (9).

$$
\left\|\mathbf{D}_{i, j}^{* n}-\mathbf{D}_{i, j}^{*(n-1)}\right\|<\varepsilon
$$

where $\varepsilon$ is the upper bound of iterative error. The number of iterations required to achieve the optimization approximation depends on this stopping criteria. In this algorithm, the error analysis showed that 500 iterations are enough to satisfy the stopping criteria $\varepsilon<0.007$.

\section{APPLICATION OF RADIAL BASIS FUNCTIONS}

In this paper, we propose to incorporate radial basis function (RBF) with the Demons algorithm. The deformation matrix $\mathbf{D}$ can be approximated by RBF scheme. The basic idea of the radial basis function interpolation is to approximate the unknown displacement function, $\{\mathbf{D}(\mathbf{x}): \mathbf{x} \in$ $\mathbb{R}^{d}$ \} by a RBF interpolant at a set of $\mathrm{N}$ distinct nodes.

Let $\left(\varphi: \mathbb{R}^{+} \rightarrow \mathbb{R}\right)$ be a basis function and $\left(\Phi_{j}: \mathbb{R}^{d} \rightarrow \mathbb{R}\right)$ is defined as in (10).

$$
\Phi_{j}(\mathbf{x})=\varphi\left(\left\|\mathbf{x}-\widetilde{\mathbf{x}_{J}}\right\|_{2}\right), \quad \mathbf{x}, \widetilde{\mathbf{x}_{J}} \in \mathbb{R}^{d} .
$$

Here, $\varphi\left(\left\|\mathbf{x}-\widetilde{\mathbf{x}_{j}}\right\|_{2}\right)$ is a typical type of RBF solely dependent on the Euclidean distance $\left\|\mathbf{x}-\widetilde{\mathbf{x}_{J}}\right\|_{2}$ between $\mathbf{x}$ and a fixed point $\widetilde{\mathbf{x}}_{J} \in \mathbb{R}^{d}$.

The basic concept of the radial basis function (RBF) method is to interpolate the displacement function $\mathbf{D}(\mathbf{x})$ using a finite series of radial basis functions at $N$ given distinct points $\mathbf{x}_{j}$ as expressed in (11).

$$
\mathbf{D}(\mathbf{x}) \approx \widetilde{\mathbf{D}(\mathbf{x})}=\sum_{J=1}^{N} \alpha_{j} \varphi\left(\left\|\mathbf{x}-\widetilde{\mathbf{x}}_{J}\right\|_{2}\right)
$$

where $\mathbf{x}, \widetilde{\mathbf{x}_{j}} \in \mathbb{R}^{d}$, and $\left\{\alpha_{j}: j=1,2, \cdots, N\right\}$ are the unknown coefficients to be determined by collocating $\widetilde{\mathbf{D}\left(\mathbf{x}_{\mathrm{k}}\right)}=\mathbf{D}\left(\mathbf{x}_{\mathrm{k}}\right)$ at a set of distinct points $\mathbf{x}_{\mathrm{k}} \subseteq \mathbb{R}^{\mathrm{d}}$, for $\mathrm{k}=1,2, \ldots, \mathrm{N}$ over the domain $\Omega$. The finite series in equation (11) yields the system of linear equations which can be expressed in matrix form as in (12).

$$
\left[\mathbf{A}_{\varphi}\right][\boldsymbol{\beta}]=[\mathbf{D}],
$$

where $[\boldsymbol{\beta}]=\left[\alpha_{1}, \alpha_{2} \cdots, \alpha_{N}\right]^{\mathrm{T}}$ are the unknown coefficients to be determined and [D] is an $\mathrm{N} \times 1$ column vector as in (13).

$$
[\mathbf{D}]=\left[\mathrm{D}\left(\mathbf{x}_{1}\right), \mathrm{D}\left(\mathbf{x}_{2}\right), \cdots, \mathrm{D}\left(\mathbf{x}_{\mathbf{N}}\right)\right]^{T} \text {. }
$$

The matrix $\left[\mathbf{A}_{\varphi}\right]$ is an $\mathrm{N} \times \mathrm{N}$ coefficient matrix as given in (14).

$$
\left[\mathbf{A}_{\varphi}\right]=\left[\begin{array}{ccc}
\varphi\left\|\left(\mathbf{x}_{1}-\mathbf{x}_{1}\right)\right\| & \ldots & \varphi\left\|\left(\mathbf{x}_{1}-\mathbf{x}_{\mathrm{N}}\right)\right\| \\
\varphi\left\|\left(\mathbf{x}_{2}-\mathbf{x}_{\mathbf{j}}\right)\right\| & \ldots & \varphi\left\|\left(\mathbf{x}_{2}-\mathbf{x}_{\mathrm{N}}\right)\right\| \\
\vdots & \ldots & \vdots \\
\vdots & \ldots & \vdots \\
\varphi\left\|\left(\mathbf{x}_{\mathrm{N}}-\mathbf{x}_{1}\right)\right\| & \ldots & \varphi\left\|\left(\mathbf{x}_{\mathrm{N}}-\mathbf{x}_{\mathrm{N}}\right)\right\|
\end{array}\right]
$$

for $i, j=1,2, \ldots, N$. To ensure that the coefficient matrix $\left[\mathbf{A}_{\varphi}\right]$ is invertible, all interpolation points belonging to the interior and boundaries in $\Omega$ must be distinct. Provided that the chosen radial basis function $\varphi$ is positive definite, the matrix $\left[\mathbf{A}_{\varphi}\right]$ is non-singular so the linear system in (12) has a unique solution. The unknown coefficients vector $[\boldsymbol{\beta}]$ can be obtained uniquely by solving the linear system by Gaussian elimination method as in (15).

$$
[\boldsymbol{\beta}]=\left[\mathbf{A}_{\varphi}\right]^{-1}[\mathbf{D}]
$$

A general theory on the existence, uniqueness and convergence of the RBF interpolation was proven by Micchelli [8] in 1986. Later, Powell [9] and Madych et al [10] extended the study and deduced the important non-singularity properties of the RBF interpolation. Their analysis proved that the RBF interpolation methods hold a truly mesh-free algorithm and a super-convergent property. The accuracy of the RBF interpolant has an order of convergence $\mathrm{O}\left(h^{d+1}\right)$, where $h$ is the density of the collocation points and $d$ is the spatial dimension.

The most popular types of radial basis functions are those given in (16).

$$
\varphi\left(r_{j}\right)=\left\{\begin{array}{cc}
\left(r_{j}^{2}\right) \log \left(r_{j}\right), & \text { Thin plate splines in } \mathbb{R}^{2} \\
e^{-\mu r_{j}^{2}}, & \text { Gaussian, } \mu>0 \\
\left(r_{j}^{2}+\delta^{2}\right)^{\frac{1}{2}}, & \text { Multiquadric, } \delta^{2} \in \mathbb{R} \\
1 /\left(r_{j}^{2}+\delta^{2}\right)^{\frac{1}{2}}, & \text { Reciprocal Multiquadric, } \delta^{2} \in \mathbb{R}
\end{array}\right.
$$

where $\left\{r_{j}=\left\|\mathbf{x}-\widetilde{\mathbf{x}}_{j}\right\|, j=1,2, \cdots, N\right\}$ is the Euclidean distance between $\mathbf{x}$ and a fixed point $\widetilde{\mathbf{x}_{J}} \in \mathbb{R}^{d}$, and $\delta^{2} \in \mathbb{R}$ is called the shape parameter in multiquadric functions. This shape parameter is used to control the fitting of a smooth surface to the data.

\section{Case Study of Prostate Registration Image}

In order to illustrate how the proposed model is applied in real-life systems, a deformable image registration from a patient with prostate cancer is used as a reference case alongside with the study. The original patient image and the deformed CT are showed in Fig. (2) (a) and (b) respectively.
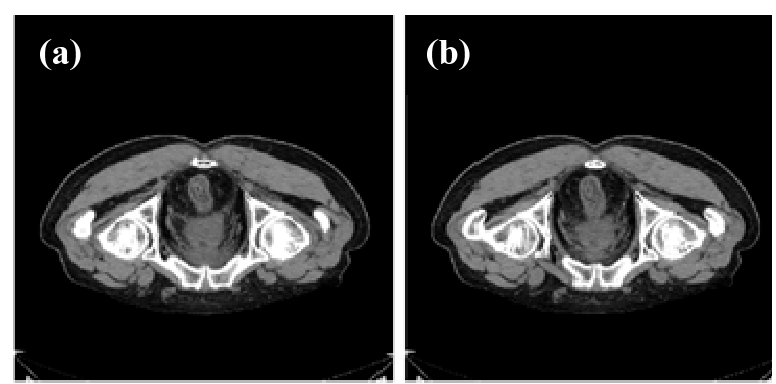

Fig. 2. (a) the original patient's image, (b) the deformed CT

Remark: Local ethics committee approval was obtained for a waiver of informed consented for retrospective analysis of 30 Intensity Modulated Radiation Therapy (IMRT) plans delivered between January 2005 and January 2006 in the Department of Clinical Oncology from a collaborative hospital. The original geometry background contains $512 \times 512$ 
pixels. In the computation, the target region is formed by removing the insignificant backgrounds which include air with intensity 0 , and bone with intensity 1 . After removing the insignificant pixels, a total of 79,743 valid pixels are remained in the target region. The input data points for computations will be based on these valid pixels. To demonstrate the performance of the proposed methods, the computed results at several different image levels are computed.

\section{COMPUtational Results}

The computational results presented in this paper are validated against a real deformable image registration for radiation therapy of prostate cancer. In the numerical examples, the input data of reference image $\mathbf{s}$ and the initial moving image $\mathbf{m}$ are taken from the image \#60 and image \#50 respectively. The image \#60 consists of 72,501 non-zero gradient pixels and 7,242 zero gradient pixels.

\section{A. By Iterative Formula of Demons Algorithm}

The Demons algorithm introduced previously in (4) and (5) are applied to this case study. Using the given data from $\mathbf{S}$ and $\mathbf{m}$, the transformation matrix $\mathbf{D}$ is generated. The corresponding registered image can then be approximated by using the displacement $\mathbf{D}$.

The gradients of static image $\nabla \mathbf{s}_{x}^{0}$ and $\nabla \mathbf{s}_{\boldsymbol{y}}^{0}$ defined in (7) and (8) are coefficient matrices computed by using the original static image. The numerical scheme of the Demons algorithm is based on the iterative scheme. The Gaussian filter is applied to smooth the result in each of iteration.

The stopping criterion is set at $\varepsilon=0.007$. The optimum result can be achieved in 500 iterations. The root mean square error is about 0.071 to 0.072 . The rate of convergence of Thirion and Cachier Demons algorithm are compared as depicted in Fig. 3.

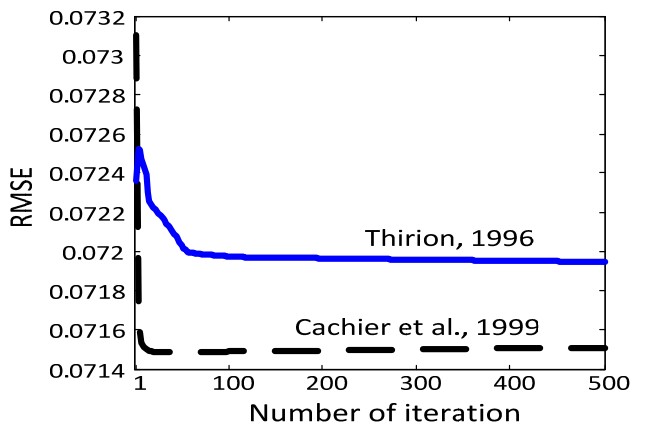

Fig. 3. The rate of convergence of two demons algorithm are compared

The computed registered image is compared with the reference image in Fig. 4 (a) and (b).
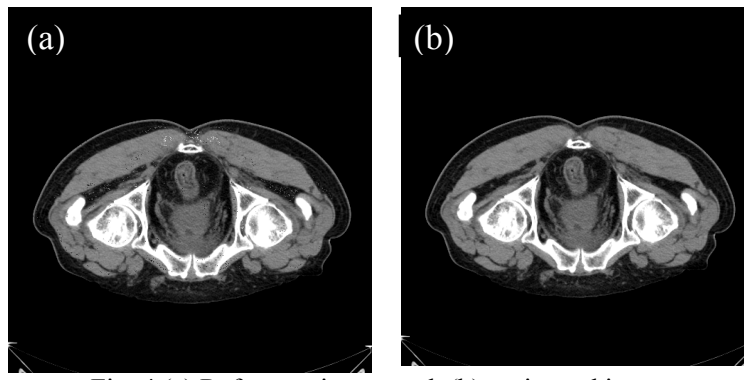

Fig. 4 (a) Reference image and (b) registered image

\section{B. By Radial Basis Function Approximation}

In the application of radial basis function method, the choice of collocation points is an important consideration. In this numerical example, the collocation points are choice in every two pixels with non-zero gradient images. A total of 17,908 distinct collocation points are selected over the target domain of the reference image \#60.

The deformation matrix $\mathbf{D}(\mathbf{x})$ in (4) is approximated by the radial basis function method as in (17).

$$
\sum_{i=1}^{N} \alpha_{i} \varphi^{n}\left(\mathrm{r}_{i, j}\right)=\sum_{i=1}^{N} \alpha_{i} \varphi^{n-1}\left(\mathrm{r}_{i, j}\right)-\frac{\left(\mathbf{m}_{i, j}^{n-1}-\mathbf{s}_{i, j}^{0}\right) \nabla \mathbf{s}_{i, j}^{0}}{\left|\nabla \mathbf{s}_{i, j}^{0}\right|^{2}+\left|\left(\mathbf{m}_{i, j}^{n-1}-\mathbf{s}_{i, j}^{0}\right)\right|^{2}}
$$

where $i, j=\left\|x_{j}-x_{j}\right\|$, for $\mathrm{i}, \mathrm{j}=1,2,3, \cdots, \mathrm{N}$. The system of (17) is subject to the following initial conditions:

$$
\left\{\begin{array}{c}
\mathbf{D}_{i, j}^{0} \cong \sum_{j=1}^{N} \alpha_{j} \varphi^{0}\left(\mathrm{r}_{k}\right)=\mathbf{0} \\
\mathbf{m}_{i, j}^{0}=\widehat{\mathbf{m}}_{i j} \\
\mathbf{s}_{i, j}^{0}=\widehat{\mathbf{s}}_{i j},
\end{array}\right.
$$

where $\widehat{\mathbf{m}}_{i j}$ and $\hat{\mathbf{s}}_{\boldsymbol{i} j}$ are the intensities of the choose collocation points from the original static $\mathbf{s}$ and move images m. The gradients $\nabla \mathbf{s}_{i, j}^{0}$ of the static image will be also be approximated by RBF as in (18).

$$
\nabla \mathbf{s}^{\mathbf{0}}(x, y)=\left(\sum_{i=1}^{N} \beta_{i} \frac{\partial \varphi_{s^{0}}\left(\mathrm{r}_{i, j}\right)}{\partial x}, \sum_{i=1}^{N} \beta_{i} \frac{\partial \varphi_{s^{0}}\left(\mathrm{r}_{i, j}\right)}{\partial y}\right) .
$$

The unknown coefficients $\beta_{j}$ are determined by collocating $\widetilde{\mathbf{s}\left(\mathbf{x}_{\mathrm{j}}\right)}=\mathbf{s}\left(\mathbf{x}_{\mathrm{j}}\right)$ at the set of distinct points $\mathbf{x}_{\mathrm{j}}$, for $\mathrm{j}=1,2, \ldots, \mathrm{N}$ over the reference domain.

This study adopted the reciprocal Multiquadric function (RMF) as the basis function because it has a super-convergent property. The smoothness of approximation can be improved by taking the normalized form as in (19).

$$
\varphi\left(r_{i, k}\right)=\frac{1}{\left[\left(\frac{\left(x_{i}-x_{k}\right)^{2}}{511^{2}}+\frac{\left(y_{i}-y_{k}\right)^{2}}{511^{2}}\right)+\delta^{2}\right]^{\frac{1}{2}}} .
$$

The numerical results have been analyzed with different shape parameter $\delta$. It was found that the optimal result can be achieved by taking the shape parameter $\delta$ between $0.5 \leq \delta \leq$ 1.

Following the Demons algorithm, the corresponding registered image $\mathbf{m}$ in the $n^{\text {th }}$ iteration can be computed according to equation (6). The Root-Mean-Square error is computed to quantify the difference between the static image and the registered image. The formula of Root-Mean-Square error is taken as in (20).

$$
\left\|\sum_{i=1}^{N} \alpha_{i} \varphi^{n}\left(r_{i, j}\right)-\sum_{i=1}^{N} \alpha_{i} \varphi^{n-1}\left(r_{i, j}\right)\right\|<\varepsilon,
$$

where $\varepsilon$ is the upper bound of iterative error. The static image and the computed registered image are compared in Fig. 5(a) and (b) respectively. 

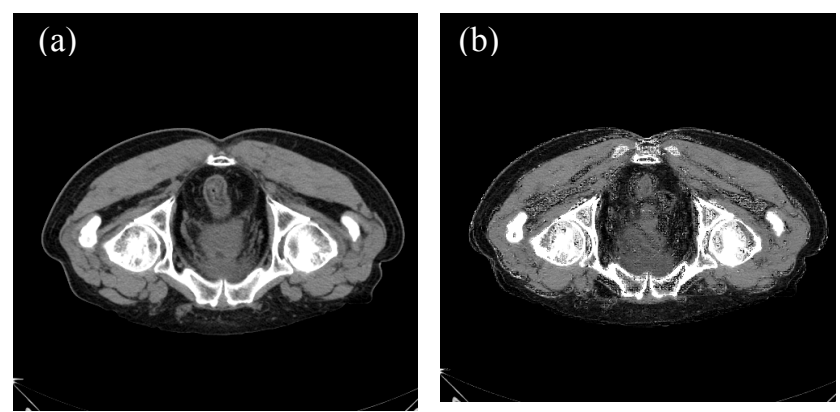

Fig. 5. (a) static image and (b) registered image.

The approximated registered image $\mathbf{m}$ are compared with results obtained by the classical iterative Demon's algorithm that purposed by two researchers, Thirion [6] in 1996 and Cachier [7] in 1999. The root mean square errors (RMSE) of different methods are summarized in Table I.

TABLE I: COMPARISON WITH ROOT MEAN SQUARE ERROR (RMSE)

\begin{tabular}{ll}
\hline \hline Methods & RMSE \\
\hline RMQ & 0.0244 \\
Thirion, 1996 & 0.0719 \\
Cachier, 1999 & 0.0715 \\
\hline \hline
\end{tabular}

The computed deformation grid by RBF methods is shown in Fig. 6(a). The difference between the registered image and moving image is shown in Fig. 6(b).

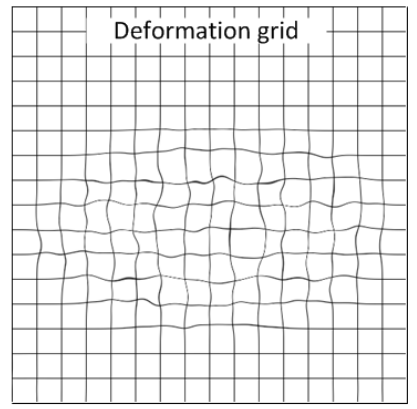

(a)

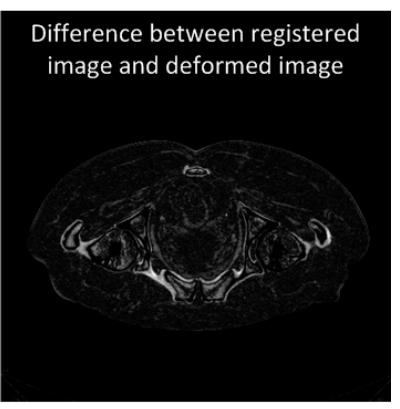

(b)
Fig. 6. (a) Computed deformation grid and (b) difference between registered image and moving image

The proposed RBF scheme produced a high degree of accuracy as compared to that obtained using the classical Demon's algorithm. However, the computation time has been shown to be more expensive due to the shortcoming of the global RBF effect in compared with the Demon's algorithm.

\section{CONCLUSION}

This study developed a meshless computational scheme based on radial basis functions to incorporate with the classical Demons algorithm. The proposed scheme was applied to a real-life deformable image registration from a patient suffered from prostate cancer. The computed registration results using the reciprocal Multiquadric RBFs and the classical Demon's algorithm were compared. The numerical experiments were shown to be with small RMSE using RBF methods which demonstrated the applicability of our proposed approach to registering pre-operative images (reference image) with post-operative image (moving image).
Further investigations on the computational efficiency and clinical applicability are necessary to determine appropriate local basis function as well as support sizes of the RBFs.

\section{ACKNOWLEDGMENT}

The project is sponsored by the President's Advisory Committee on Research and Development. Project Number: 2010/2.1.

\section{REFERENCES}

[1] C. Broit, "Optimal registration of deformed images," Ph.D. Dissertation, Computer and Information. Science Dept., University of Pennsylvania, 1981.

[2] T. Netsch, P. Roesch, A. van Muiswinkel, and J. Weese, "Towards real-time multimodality $3 \mathrm{~d}$ medical image registration," in Proc. 8th Int. Conf. Comput. Vision (Vancouver, Canada, 2001, 2006).

[3] F. L. Bookstein. Principal Warps, "Thin-Plate Splines and the Decomposition of Deformations," IEEE Trans. Pattern Anal. Mach. Intell., vol. 11, no. 6, pp.567-585, 1989.

[4] R. L. Hardy, "Multiquadric equations of topography and other irregular surfaces," J. Geophys, Res, vol. 176, pp. 1905-1915, 1971.

[5] E. M. V. Osorio, M. S. Hoogeman, L. Bondar, P. C. Levendag, and B. J. M. Heijmen, "A novel flexible framework with automatic feature correspondence optimization for non-rigid registration in radiotherapy," Medical Physics, vol. 36, no. 7, pp.2848-2859, 2009.

[6] J. P. Thirion, "Non-rigid matching using demons," in Proc. Conf. Computer Vision and Pattern Recognition, pp. 245-251, June 1996.

[7] X. Pennec, P. Cachier, and N. Ayache, "Understanding the demon's algorithm: 3D non-rigid registration by gradient descent," in Proceedings of International Conference on Medical Image Computing and Computer-Assisted Intervention, vol. 1679 of LNCS. Springer-Verlag, Berlin, pp. 597-605, MICCAI 1999.

[8] C. A. Micchelle, "Interpolation of scattering data: distance matrices and conditionally positive definite functions," Constr. Approx. 2. pp. 11-22, 1986

[9] M. J. D. Powell, "Wavelets, subdivision algorithms and radial basis functions," The theory of radial basis functions approximations in 1990, Ch. 3, ed. Will Light, Vol. II, Oxford University Press, pp. 105-210, 1990.

[10] W. R. Madych and S. A. Nelson, "Multivariate interpolation and conditionally positive definite functions", Approx. Theory Appl., vol. 4, pp. 77-89, 1988.

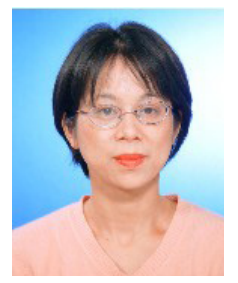

Sze Mui Wong received a BSc (Hons) degree in Mathematical Studies in 1988, and received an MSc degree in Operational Research in 1989 at the University of London. She completed the $\mathrm{PhD}$ in Computational Modeling in 2001 in the City University of Hong Kong. She joined the Open University of Hong Kong in 1993 as a lecturer. She is now an associate professor in the same university.

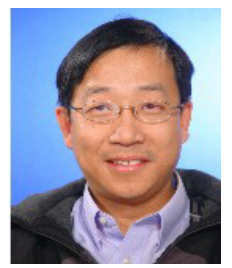

Tak Sing Li received a BSc (Hons) degree in mechanical engineering from the University of Hong Kong. He then received an MSc degree in computer science in 1991 and a $\mathrm{PhD}$ in computer science in 1994. Both degrees were from the Queen's University of Belfast. He joined the Open University of Hong Kong in 1995 as a lecturer in the computing team. He is now an associate professor in the same university.

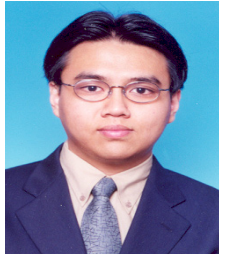

Kei Shing Ng received a BSc (Hons) degree in Computational Mathematics in 2001, and received an MPhil Degree in Applied Mathematics from the City University of Hong Kong in 2003 and a $\mathrm{PhD}$ in Medical informatics in 2009 from Hong Kong Polytechnic University. He joined the Open University of Hong Kong in 2011 as a lecturer. 